\title{
Avez-vous un diplôme de formation continue de I'ISFM?
}

\section{Christoph Hänggeli}

Directeur de I'ISFM

\section{La formation continue: un devoir profes- sionnel légal}

Pour toutes les cinq professions médicales universitaires, l'obligation de suivre une formation continue est prévue explicitement en tant que devoir professionnel dans la loi sur les professions médicales (LPMéd). Les autorités cantonales chargées de la surveillance peuvent sanctionner tout manquement au devoir de formation continue par un avertissement ou une amende pouvant aller jusqu'à 20000 francs. En revanche, la mise en œuvre de ce devoir légal, notamment son étendue et ses modalités, incombe aux organisations professionnelles. Conjointement avec les sociétés de discipline médicale, l'ISFM propose au corps médical un diplôme de formation continue reconnu de la même façon par les autorités sanitaires et les assureurs-maladie. Il existe dans chaque domaine (titres de spécialiste uniquement) un programme de formation continue qui règle en détail les conditions d'obtention du diplôme. Le choix parmi les 46 programmes de formation continue relève de la responsabilité de chacun. Il est recommandé de choisir un programme qui correspond le plus à l'activité professionnelle principale du moment. Le devoir de formation continue commence dans l'année suivant l'obtention du titre de spécialiste ou le début de l'activité médicale en Suisse. Les médecins qui se trouvent en formation postgraduée en vue d'un titre de spécialiste ou de formation approfondie ne sont pas soumis à cette exigence.

\section{Résumé}

La toute nouvelle plate-forme de formation continue en ligne de I'ISFM permet aux médecins de saisir encore plus facilement la formation suivie et de la confirmer grâce au diplôme de formation continue. Les sociétés de discipline médicale contrôlent les informations saisies en ligne, puis approuvent l'impression du diplôme. Les diplômes de formation continue sont ensuite automatiquement publiés sur www.doctorfmh.ch, ce qui rend obsolète toute attestation individuelle vis-à-vis des autorités sanitaires et des assureurs-maladie. Les assureurs continuent de rémunérer les prestations de droits acquis aux médecins dont le diplôme de formation continue est publié sur www.doc torfmh.ch.

Grâce à la plate-forme de formation continue, la charge administrative est réduite au strict minimum à la fois pour les médecins soumis au devoir de formation continue et pour les sociétés de discipline.

\section{Extension de la plate-forme}

Les fonctions de la plate-forme de formation continue en ligne vont être élargies. Un registre centralisé des sessions de formation permettra notamment de simplifier la recherche de sessions. Par ailleurs, les médecins pourront inscrire la session dans leur protocole directement lors de la manifestation en scannant le code $\mathrm{QR}$ à disposition sur place avec leur téléphone portable. Les sociétés et les organisateurs pourront également y annoncer leurs manifestations et attirer davantage de participants.

\section{Formation continue essentielle spécifique et formation continue élargie}

Le nombre d'heures de formation continue conseillé par an (cf. fig. 1) est de 80 heures (= crédits). Les médecins doivent attester 50 crédits par an ou 150 crédits au cours d'une période de formation continue de trois ans. Chaque programme définit une formation continue essentielle spécifique de 25 crédits par an. Les sociétés de discipline médicale peuvent définir différentes catégories de formation et fixer le nombre maximal de crédits acceptés par catégorie. Les médecins peuvent faire valider 25 crédits au plus en tant que formation continue élargie. On entend par formation continue élargie toutes les sessions de formation pour lesquelles des crédits sont octroyés par une autre société de discipline médicale, une société cantonale de médecine ou l'ISFM. Cela permet aux médecins de suivre une formation continue hors de leur discipline principale. Les questions relatives au devoir de formation continue dans une discipline précise doivent être adressées à la société concernée.

\section{Comment puis-je obtenir un diplôme de formation continue?}

Tout simplement sur la plate-forme de formation continue de l'ISFM (www.siwf.ch $\rightarrow$ Formation continue; cf. fig. 2). Cette plate-forme vous permet d'enregistrer continuellement vos activités dans un protocole personnel et d'imprimer vous-même votre diplôme au terme de la période de formation continue de trois ans - à condition bien sûr d'avoir obtenu les 150 crédits exigés par le programme choisi. Tous les diplômes obtenus par le biais de la plate-forme apparaissent automatiquement dans le registre officiel www.doctorfmh.ch. Les médecins qui ne 


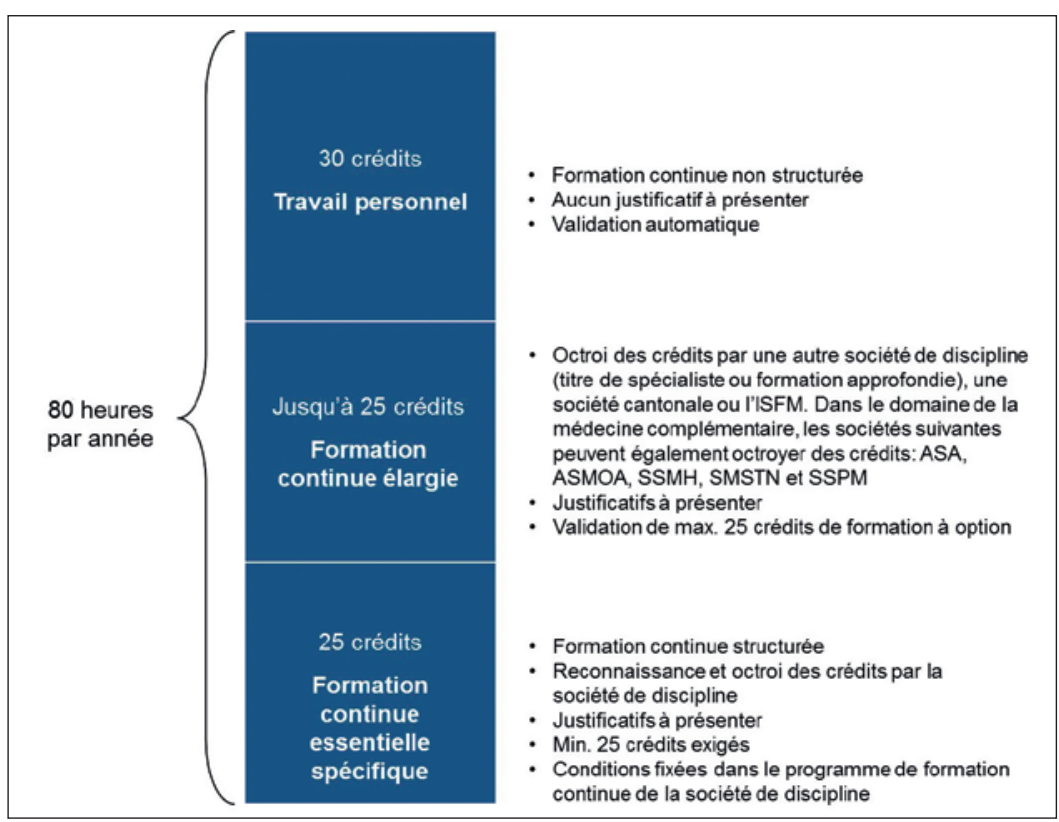

Figure 1: Structure des 80 heures de formation continue exigées par année.

disposent pas du titre de spécialiste correspondant obtiennent une attestation équivalente certifiant à l'intention des autorités sanitaires et des assureurs-maladie que le devoir de formation continue a été accompli.

\section{Pas de rémunération des prestations de droits acquis sans diplôme de formation continue!}

Treize ans après l'entrée en vigueur du TARMED, quelque 7800 médecins font encore valoir 510000 prestations de droits acquis. Pour pouvoir continuer à les facturer, ils doivent attester leur formation continue au moyen d'un diplôme approprié. Pour cela, ils disposent des programmes de formation continue des 46 titres de spécialiste. Grâce à la formation continue élargie, chaque programme offre la possibilité de suivre une formation continue dans les disciplines de chacune des positions sollicitées. Ces positions sont automatiquement validées et prolongées tout au long de la durée de validité du diplôme de formation continue.
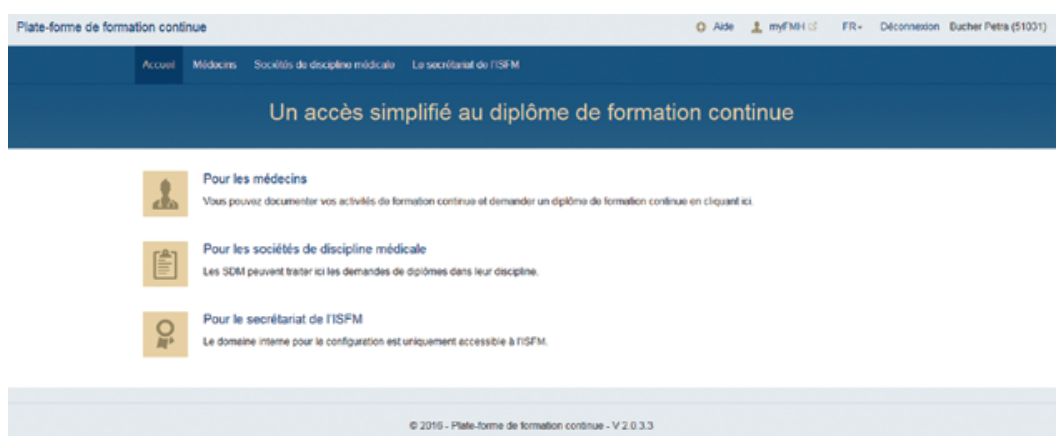

Figure 2: Page d'accès à la plate-forme de formation continue.

\section{Conclusion et perspectives}

Pour les médecins en exercice, suivre une formation continue tout au long de leur carrière est une évidence après leur formation postgraduée. La plate-forme en ligne de l'ISFM et le diplôme de formation continue leur permettent d'attester sans autre formalité que leurs compétences professionnelles sont à jour. Le diplôme de formation continue a deux fonctions:

- prouver que le devoir légal de formation continue a été accompli et

- valider toutes les positions de droits acquis.

Le diplôme de formation continue sera-t-il exigé à l'avenir pour l'autorisation générale de facturer et pour l'autorisation de facturer à la charge de l'assurance obligatoire des soins? Ces deux mesures sont actuellement en discussion et montrent que le diplôme de formation continue représentera à l'avenir la référence dans ce domaine. Pourquoi donc ne pas s'inscrire dès à présent sur la plate-forme?

\section{La formation continue en 10 points}

1. Le devoir de formation continue est un devoir professionnel inscrit à l'art. 40 de la LPMéd. La surveillance et les contrôles incombent aux autorités cantonales concernées ou (par délégation) aux sociétés cantonales de médecine.

2. L'ISFM offre un diplôme de formation continue conjointement avec les 46 sociétés de discipline médicale représentant un titre de spécialiste. Ce diplôme permet

- de prouver que le devoir légal selon la LPMéd a été accompli et

- de valider toutes les positions de droits acquis.

3. Conformément à la Réglementation pour la formation continue (RFC), les médecins doivent obtenir au moins un diplôme de formation continue dans le domaine correspondant à leur activité professionnelle principale du moment.

4. Chaque programme de formation continue des 46 sociétés de discipline médicale fait la distinction entre les catégories suivantes:

- formation continue essentielle ( 25 crédits par an, contrôlés)

- formation continue élargie (25 crédits par an, contrôlés)

- travail personnel (30 heures par an, non contrôlées) La formation continue obligatoire comprend donc 80 heures par an. Les médecins en activité doivent attester 150 crédits (dont au moins 75 crédits de formation continue essentielle) tous les trois ans.

5. Les médecins qui facturent des positions de droits acquis dans le cadre du TARMED doivent suivre 
Diplômes de formation continue dans le registre des médecins www.doctorfmh.ch

Le registre des médecins www.doctorfmh.ch contient, outre toutes les qualifications professionnelles officielles selon la Réglementation pour la formation postgraduée (RFP), également les diplômes de formation continue avec leur période de validité. En revanche, il ne contient pas d'autres informations, par exemple concernant les sessions enregistrées dans le protocole, afin de garantir la protection des données.

une formation continue aussi dans ces domaines, conformément au concept de valeur intrinsèque. La formation continue pour ces positions peut être accomplie dans le cadre de la formation continue élargie avec 75 crédits sur une période de 3 ans.

6. Si vous n'obtenez pas (ne souhaitez pas obtenir) de diplôme de formation continue, vous avez l'obligation de déclarer la formation continue pour les prestations de droits acquis sur www.myfmh.ch, et ce pour chaque position.

7. Le diplôme de formation continue doit être renouvelé tous les 3 ans.

8. La recertification (formation continue) exigée pour certaines attestations de formation complémentaire est indépendante des 46 diplômes de formation continue des titres de spécialiste. La non-observation des règles de recertification peut entraîner la perte de l'attestation. Il en va de même des formations approfondies interdisciplinaires.

9. Vous trouverez sur notre site Internet www.siwf.ch, à la rubrique "Formation continue», de plus amples informations sur les 46 programmes de formation continue et les coordonnées des interlocuteurs des sociétés de discipline médicale.

10. La plate-forme de formation continue (www.siwf.ch $\rightarrow$ Formation continue $\rightarrow$ Plate-forme de formation continue) vous permet d'enregistrer continuellement vos activités et d'imprimer vous-même le diplôme de formation continue après l'obtention des 150 crédits exigés.

\section{Questions fréquentes}

\section{Vais-je perdre mon titre de spécialiste si je ne suis pas de formation continue?}

Non, la formation continue est un devoir professionnel dont le manquement peut être sanctionné par les autorités sanitaires cantonales par un avertissement ou une amende pouvant aller jusqu'à 20000 francs. Le retrait du titre de spécialiste n'est pas possible.

\section{Qui a l'obligation d'accomplir la formation continue?} En vertu de l'art. 9 de la Réglementation pour la formation continue (RFC), tous les détenteurs d'un titre postgrade fédéral (aussi celui de «Médecin praticien») ou d'un titre postgrade étranger reconnu sont tenus d'accomplir une formation continue aussi longtemps qu'ils exercent une activité médicale en Suisse. Les médecins qui se trouvent en formation postgraduée en vue d'un titre de spécialiste ou de formation approfondie ne sont pas soumis à cette exigence, y compris les médecins en formation qui possèdent déjà un titre de formation postgraduée.

\section{Je me rends aux Etats-Unis pour travailler deux ans dans la recherche. Suis-je tenu d'accomplir une formation continue?}

Non, vous n'êtes pas tenu de suivre de formation continue pendant cette période. Le devoir de formation continue s'applique uniquement aux médecins exerçant une activité médicale en Suisse (art. 9 RFC) et ne reprendra vigueur qu'à votre retour en Suisse. Vous pourrez alors soit commencer une nouvelle période de formation continue de trois ans, soit faire valoir une réduction de deux ans de la formation continue obligatoire pour votre séjour à l'étranger. Il faut préciser que les séjours de courte durée à l'étranger (inférieurs à quatre mois) ne suffisent pas pour suspendre le devoir de formation continue.

\section{Je travaille dans le secteur administratif de} Swissmedic en tant que médecin porteur d'un titre de spécialiste en médecine interne générale. Suis-je tenu d'accomplir une formation continue?

Le devoir de formation continue concerne tous les médecins qui exercent une «activité médicale». Une telle activité a lieu lorsque vous examinez, conseillez ou soignez des patients sous une forme quelconque. Une activité administrative ou de recherche sans contact avec des patients n'entre pas dans cette catégorie.

\section{Que se passe-t-il si je n'obtiens pas de diplôme de formation continue?}

Contrairement au devoir de formation continue, l'obtention du diplôme de formation continue n'est pas une obligation stipulée par la loi. L’important est que vous accomplissiez la formation continue prescrite. Cela ne concerne en aucun cas votre titre de spécialiste. Ne pas posséder de diplôme de formation continue entraîne toutefois les inconvénients suivants:

- En cas de contrôle, vous devez convaincre les autorités cantonales concernées que la formation continue accomplie correspond au standard habituel. Il en va de même lors d'un procès éventuel en responsabilité civile.

- En l'absence de diplôme inscrit dans le registre www.doctorfmh.ch, vous devez confirmer dans la banque de données (www.myfmh.ch) la formation 
continue spéciale pour les prestations de droits acquis et l'attester au moyen de documents adéquats. Si vous ne le faites pas, les assureurs peuvent refuser la prise en charge des positions de droits acquis.

- A l'avenir, le diplôme de formation continue pourrait être exigé pour l'autorisation générale de facturer et pour l'autorisation de facturer à la charge de l'assurance obligatoire des soins.

6. Quel est le coût d'un diplôme de formation continue? La reconnaissance de sessions de formation continue et le contrôle de la formation continue sont effectués par la société de discipline médicale concernée, laquelle perçoit des taxes en fonction des dépenses occasionnées. Pour les membres de la société, cette taxe est généralement incluse dans la cotisation annuelle. Pour les non-membres, le diplôme valable pour trois ans ne devrait pas coûter plus de 300 francs. Les taxes plus élevées doivent être approuvées par le Comité de l'ISFM.

\section{Comment puis-je accomplir une formation continue dans le cadre de la médecine complémentaire si ma société ne reconnaît aucune session de formation correspondante?}

Dans la Réglementation pour la formation continue (RFC), la médecine complémentaire fait l'objet d'une disposition spéciale: les cinq sociétés qui gèrent une attestation de formation complémentaire (ASA, ASMOA, SSMH, SMSTN, SSPM) peuvent reconnaître des sessions de formation et octroyer des crédits correspondants qu'il est possible de faire valider dans le cadre de la formation continue élargie.

\section{Puis-je faire valoir la formation continue que j'accomplis dans le cadre d'une attestation de formation complémentaire également pour le diplôme de formation continue?}

Seules les sessions de formation pour lesquelles des crédits sont octroyés par une société de discipline médicale (titre de spécialiste), une société cantonale de médecine, les cinq sociétés de médecine complémentaire (ASA, ASMOA, SSMH, SMSTN, SSPM) ou l'ISFM peuvent être prises en compte pour le diplôme de formation continue. Les sessions de formation d'autres organisations médicales doivent être reconnues au moins par une société de discipline médicale. Lorsque c'est le cas, ces sessions peuvent être prises en compte dans le cadre de la formation continue élargie pour les 46 autres diplômes de formation continue.

9. Je suis porteur du titre de spécialiste en médecine interne générale et j'exerce principalement dans le domaine gynécologique. Je dispose des positions de droits acquis correspondantes. Puis-je obtenir le diplôme de formation continue de la Société suisse de gynécologie et d'obstétrique (SSGO) et attester ainsi la formation continue pour les prestations de droits acquis?

Comme vous exercez principalement en gynécologie et obstétrique, vous avez intérêt à choisir le programme de formation continue de la SSGO. Mais en vertu de l'art. 12 de la RFC, le diplôme de formation continue n'est remis qu'aux porteurs du titre correspondant. Si vous validez 150 crédits, vous avez droit à une attestation de formation continue équivalente qui vous servira entre autres à attester la formation continue requise pour les droits acquis.

\section{Je possède deux titres de spécialiste, l'un en médecine interne générale et l'autre en cardiologie. Dois-je obtenir un diplôme de formation continue dans ces deux domaines?}

Non. Vous pouvez vous limiter au programme de formation continue qui correspond le plus à l'activité professionnelle que vous exercez. Vous êtes bien évidemment libre de suivre deux programmes de formation continue. Cette double formation n'entraîne pas une importante surcharge de travail, puisque la formation continue essentielle d'un des programmes est automatiquement considérée comme la formation continue élargie de l'autre. Dès que vous avez accompli les formations continues essentielles spécifiques des deux disciplines sans chevauchement, vous avez droit aux deux diplômes de formation continue.

11. Je suis porteur d'un titre de spécialiste en médecine interne générale avec une formation approfondie en gériatrie. Existe-t-il un diplôme de formation continue pour cette spécialisation?

Non. Depuis la révision de la Réglementation pour la formation continue (RFC) du 26 mai 2010 , seuls 46 programmes de formation continue sont proposés, soit un par titre de spécialiste. Cependant, la formation continue en gériatrie est prise en compte sans limitation de contenu pour le diplôme de formation continue en médecine interne générale.

\section{Je suis mère de deux enfants et je travaille à $50 \%$} dans un cabinet médical. Dois-je accomplir l'intégralité de la formation continue?

Oui. Un taux d'occupation partiel ne donne pas droit à une réduction de la formation continue obligatoire. La formation continue a pour objectif de garantir la qualité des prestations et de maintenir les compétences médicales, une garantie qu'il convient d'apporter également lors d'une activité à temps partiel. 
13. J'ai interrompu mon activité médicale pendant un congé maternité de six mois. Dois-je valider moins de crédits pour le diplôme de formation continue?

Oui. Des interruptions de l'activité médicale dépassant quatre mois au cours de la période de formation de trois ans entraînent une réduction au prorata des crédits exigés. Ce même principe s'applique aux séjours à l'étranger ou à d'autres interruptions de l'activité médicale en Suisse. Ces motifs de réduction peuvent être sans autre saisis sur la plate-forme de formation continue.

\section{Je suis porteur du titre de "Médecin praticien".} Quelle formation continue dois-je effectuer?

Les porteurs du titre de "Médecin praticien" peuvent faire leur choix parmi les 46 domaines répertoriés. Nous leur recommandons de choisir le programme de formation continue correspondant à l'activité professionnelle exercée sur le moment.

\section{Il ne m'a pas été possible d'accomplir la formation} continue requise en trois ans. Puis-je la rattraper?

Il n'est pas possible de rattraper la formation continue l'année suivante ou de reporter des crédits sur la période de formation continue suivante. Cependant, vous pouvez en tout temps imprimer, via la plateforme de formation continue, un diplôme valable pour les trois prochaines années dès que vous avez obtenu le nombre de crédits requis (en l'absence d'un motif donnant droit à une réduction $=150$ crédits).

16. J'ai obtenu mon titre de spécialiste le 19 septembre 2016. A partir de quand suis-je tenu d'accomplir la formation continue?

Votre devoir de formation continue commence l'année qui suit celle de l'obtention de votre titre. Dans votre cas, le $1^{\text {er }}$ janvier 2017.

17. Quand puis-je détruire les attestations/justificatifs de ma formation continue? Combien de temps dois-je les conserver?

La Conférence suisse des directrices et directeurs cantonaux de la santé (CDS) et l'Association des médecins cantonaux de Suisse (AMCS) nous ont assurés que le diplôme de formation continue représentait un justificatif suffisant pour documenter le devoir de formation continue conformément à la loi sur les professions médicales (LPMéd). Toutefois, les autorités se réservent le droit d'exiger des justificatifs détaillés supplémentaires dans des cas particuliers.

Par conséquent, nous vous recommandons par sécurité de conserver ces documents. Un délai de conservation de dix ans s'applique par analogie au délai de prescription général.
18. J'ai demandé un diplôme de formation continue pour la période 2013-2015. Pourquoi ai-je reçu un diplôme valable pour la période 2016-2018? Les diplômes de formation continue sont toujours délivrés pour la période suivante et enregistrés sur www. doctorfmh.ch avec ces dates.

\section{Je suis employé comme médecin adjoint dans un} hôpital public. Le temps que je consacre à la formation continue est-il considéré comme du temps de travail? Oui. L'ordonnance 1 relative à la loi sur le travail (OLT 1, art. 3 al. 4) précise que le temps qu'un travailleur consacre à une formation continue en vertu de la loi est réputé temps de travail. Il faut partir du principe que la Réglementation pour la formation continue (RFC) de l'ISFM fait référence pour la formation continue des médecins et que 80 heures de formation continue par année sont donc considérées comme temps de travail - à condition bien évidemment que la formation continue soit effectivement suivie.

\section{Comment faire reconnaître la formation continue} suivie en France?

Selon l'art. 7 al. 2 let. c RFC, les sessions de formation continue qui obtiennent des crédits de l'institution compétente d'un Etat membre de l'UE/AELE sont automatiquement reconnues en Suisse. Toutefois, le diplôme de formation continue n'est délivré que si toutes les conditions du programme de formation continue de la discipline concernée sont remplies. Il revient à la société de discipline de décider si les crédits obtenus à l'étranger peuvent être validés pour la formation continue essentielle. On peut partir du principe qu'une session de formation en cardiologie dotée de cinq crédits en France est considérée en Suisse aussi comme formation continue essentielle en cardiologie. Dans tous les cas, une session reconnue à l'étranger devrait être validée pour la formation continue élargie sans autre formalité (jusqu’à 25 heures par an).

\section{Seuls les crédits entiers peuvent être saisis sur} la plate-forme de formation continue. Comment puisje saisir une session de 45 minutes?

Un crédit correspond à 45-60 minutes. Une session de 45 minutes ne pose donc aucun problème. Les sessions de 90 minutes donnent droit à deux crédits. En revanche, une session de 30 minutes ne peut pas être saisie en tant que telle. Dans ce cas, deux sessions d'une demi-heure donnent droit à un crédit. 